\title{
Composição corporal e capacidade cardiorrespiratória em praticantes de corrida de rua*
}

\author{
Ana Caroline Gusmão de Matos ${ }^{* *}$ \\ Antenor de Oliveira Silva Neto ${ }^{* * * *}$ \\ Ayrton Moraes Ramos ${ }^{* * * *}$ \\ Clésio Andrade Lima ${ }^{* * * * *}$ \\ Gabrielle dos Santos Moreira****** \\ Maria Eduarda da Silva Cursino Ribeiro ${ }^{\text {*********}}$ \\ Maria Luísa Barreto Paiva ${ }^{* * * * * * * * *}$ \\ Michael Douglas Celestino Bispo ${ }^{* * * * * * * * * *}$ \\ Natália Brito de Almeida \\ Estélio Henrique Martin Dantas
}

\begin{abstract}
${ }^{*}$ Artigo de pesquisa científica com autofinanciamento e vinculado ao grupo de pesquisa do Laboratório de Biociências da Motricidade Humana, Universidade Tiradentes, Aracaju, Sergipe, Brasil.

** Acadêmica de Medicina, Universidade Tiradentes, Brasil. Correio eletrônico: anagusmaao@gmail.com; ORCID: https://orcid.org/0000-0002-4451-0243

*** Mestre em Educação, professor e coordenador do curso a distância de Educação Física, Universidade Tiradentes, Aracaju, Sergipe, Brasil. Correio eletrônico: antenor oliveira@unit.br; ORCID: https://orcid.org/0000-0003-4787-7566

**** Mestre em Educação Física, docente titular de Educação Física, Universidade Tiradentes, Aracaju, Sergipe, Brasil. Correio eletrônico: ayrtonmoraes@hotmail.com; ORCID:

https://orcid.org/0000-0001-8004-3948

***** Doutor em Biotecnologia, docente titular de Educação Física, Universidade Tiradentes, Aracaju , Sergipe, Brasil. Correio eletrônico: clesio.ufs@gmail.com; ORCID: https://orcid.org/0000-00023387-9715

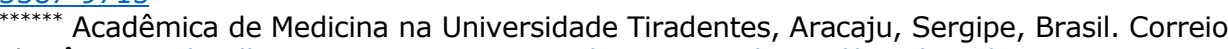
eletrônico: gabrielle.moreira@souunit.com.br; ORCID: https://orcid.org/0000-0001-5721-6898

******* Acadêmica de Medicina na Universidade Tiradentes, Aracaju, Sergipe, Brasil. Correio eletrônico: maria.cursino99@souunit.com.br; ORCID: https://orcid.org/0000-0003-1719-1563

******** Acadêmica de Medicina na Universidade Tiradentes, Aracaju, Sergipe, Brasil. Correio eletrônico: maria.Ipaiva@souunit.com.br; ORCID: https://orcid.org/0000-0001-5181-6606 Mestre em Saúde e Ambiente. Programa de Pós-graduação stricto sensu em Saúde e Ambiente da Universidade Tiradentes, Aracaju, Sergipe, Brasil. Correio eletrônico: michaeldouglasc@hotmail.com; ORCID: https://orcid.org/0000-0003-2564-1464 Acadêmica de Medicina na Universidade Tiradentes, Aracaju, Sergipe, Brasil. Correio eletrônico: natalia.brito@souunit.com.br; ORCID: https://orcid.org/0000-0003-1630-4087 ********** Doutor em Educação Física. Programa de Pós-graduação Stricto Sensu em Enfermagem e Biociências da Universidade Federal do Estado do Rio de Janeiro, Rio de Janeiro, Brasil. Programa de Pós-graduação stricto sensu em Saúde e Ambiente da Universidade Tiradentes, Aracaju, Sergipe, Brasil. Correio eletrônico: estelio henrique@unit.br; ORCID: https://orcid.org/0000-0003-0981-
\end{abstract} $\underline{8020}$

Revista de Investigación Cuerpo, Cultura y Movimiento

ISSN: 2248-4418 | e-ISSN: 2422-474X | DOI: https://doi.org/10.15332/2422474X

Vol. 11 N.o 2 | julio-diciembre de 2021 
Recibido: 03 de septiembre de 2020

Aceptado: 19 de marzo de 2021

Citar como: Matos, A. C. G., Silva Neto, A. O., Ramos, A. M., Lima, C. A., Moreira, G.

S., Ribeiro, M. E. S. C., Paiva, M. L. B., Bispo, M. D. C., Almeida, N. B., Dantas, E. H. M. (2021). Composição corporal e capacidade cardiorrespiratória em praticantes de corrida de rua. Revista de Investigación Cuerpo, Cultura y Movimiento, 11(2). https://doi.org/10.15332/2422474X.6877

(c) $\underset{\mathrm{BY}}{\mathrm{NC}} \mathrm{SA}$

\section{Resumo}

Analisar a correlação entre composição corporal e capacidade cardiorrespiratória nos praticantes de corrida de rua. Estudo quantitativo, descritivo e transversal, realizado durante o evento FastTest, em Aracaju, Brasil. O grupo amostral do estudo, selecionado de forma randômica entre os 61 participantes do evento, foi composto por 36 corredores, dos quais 21 homens ( $\underline{X}=41,86 \pm 10,4$ anos) e 15 mulheres $(\underline{X}=43,93 \pm 11,04$ anos). A coleta dos dados foi feita por meio de avaliação antropométrica (estatura, massa corporal total e dobras cutâneas) e do consumo máximo de oxigênio, pelo teste de Cooper. Foi observada correlação negativa entre percentual de gordura e VO2máx no grupo feminino $(\mathrm{r}=-0,60 ; \mathrm{p}=0,001)$ e masculino $(\mathrm{r}=-0,74 ; \mathrm{p}=0,0001)$; e entre o índice de massa corpórea e VO2máx, nos homens ( $\mathrm{r}=-0,79 ; \mathrm{p}=$ o,0001) e nas mulheres ( $\mathrm{r}=-0,63 ; \mathrm{p}=0,0005)$. Pode-se observar correlação significativa positiva entre o percentual de massa magra e o VO2máx, em homens ( $\mathrm{r}=0,74 ; \mathrm{p}=0,0001)$ e mulheres $(\mathrm{r}=0,60 ; \mathrm{p}=$ o,oo1). Evidencia-se a importância de um baixo percentual de gordura e uma maior massa magra para o desempenho físico nas modalidades aeróbicas e provas de endurance.

Palavras-chave: aptidão cardiorrespiratória, composição corporal, corrida, treino aeróbico. 


\section{Composición corporal y capacidad cardiorrespiratoria en corredores de calle}

\section{Resumen}

Analizar la correlación entre composición corporal y capacidad cardiorrespiratoria en corredores de calle. Estudio cuantitativo, descriptivo y transversal realizado durante el evento FastTest, en Aracaju, Brasil. El grupo de muestra del estudio, seleccionado aleatoriamente entre los 61 participantes del evento, estuvo compuesto por 36 corredores, de los cuales 21 participantes masculinos $(\underline{X}=41,86 \pm 10,14$ años) y 15 mujeres $(\underline{X}=43,93 \pm 11,04$ años $)$.

La recolección de datos se realizó mediante evaluación antropométrica (estatura, masa corporal total y pliegues cutáneos) y consumo máximo de oxígeno, mediante la prueba de Cooper. Se observó una correlación negativa entre el percentual de grasa y el VO2max en el grupo de mujeres $(\mathrm{r}=-0.60 ; \mathrm{p}=0.001)$ y hombres $(\mathrm{r}=-0,74 ; \mathrm{p}=0,0001) ; \mathrm{y}$ entre el índice de masa corporal y el VO2máx, en hombres $(\mathrm{r}=-0,79 ; \mathrm{p}=0,0001)$ y en mujeres $(r=-0,63 ; p=0,0005)$. Se puede observar una pendiente positiva entre el porcentaje de masa magra y el VO2max, en hombres $(r=0,74 ; p=0,0001)$ y mujeres $(r=0,60 ; p=0,001)$. Se evidencia la importancia de un bajo porcentaje de grasa y una mayor masa magra para el rendimiento físico en modalidades aeróbicas y pruebas de resistencia.

Palabras clave: capacidad cardiorrespiratoria, composición corporal, carrera, entrenamiento aeróbico.

\section{Body composition and cardiorespiratory fitness in road runners}

\section{Abstract}

To analyze the correlation between body composition and cardiorespiratory capacity in road runners. This is a quantitative, 
descriptive and cross-sectional study carried out during the FastTest event, in Aracaju, Brazil. The study sample group, randomly selected from the 61 participants of the event, was composed of 36 runners, of which 21 participants were male $(\underline{X}=41.86 \pm 10.14$ years $)$, and 15 female $(\underline{X}=43.93 \pm 11.04$ years). Data collection was made through anthropometric assessment (height, total body mass and skinfolds) and maximum oxygen consumption, using the Cooper test. A negative correlation was observed between fat percentage and VO2max was negative in the group of women $(\mathrm{r}=-0.60 ; \mathrm{p}=0.001)$ and men $(\mathrm{r}=$ $-0.74 ; \mathrm{p}=0.0001)$; and between the body mass index and VO2max, in men ( $\mathrm{r}=-0.79 ; \mathrm{p}=0.0001)$ and women $(\mathrm{r}=-0.63 ; \mathrm{p}=0.0005) . \mathrm{A}$ positive slope can be observed between the percentage of lean mass and VO2max, in men ( $\mathrm{r}=0.74 ; \mathrm{p}=0.0001)$ and women $(\mathrm{r}=0.60 ; \mathrm{p}=0.001)$. The importance of a low fat percentage and a higher lean mass for physical performance in aerobic modalities and endurance tests is evidenced.

Keywords: cardiorespiratory capacity, body composition, race, aerobic training.

\section{Introdução}

A corrida de rua despontou na Inglaterra do século XVIII e, posteriormente, alcançou outras regiões da Europa e da América do Norte. Após a primeira maratona olímpica, em 1896 na Grécia, essa modalidade do atletismo se popularizou (Perez et ál., 2004). Atualmente, as distâncias oficiais para as corridas de rua variam de $5 \mathrm{~km}$ a $100 \mathrm{~km}$, conforme determinado pela Associação Internacional das Federações de Atletismo (Ferreira et ál., 2015).

No Brasil, a São Silvestre, prova de corrida de rua que se tornou tradicional, foi disputada pela primeira vez em 1925. Desde então, a popularização dessa modalidade cresce por seus benefícios estéticos, de 
integração social ou de redução do estresse proporcionados mesmo aos corredores não profissionais (Ferreira et ál., 2015).

O desempenho nessas modalidades de longa duração depende do consumo máximo de oxigênio (VO2máx), conforme Perez et ál. (2004). Segundo o American College Sports Medicine, o VO2máx mensura o condicionamento cardiorrespiratório, sendo ele o débito cardíaco dividido pela diferença arteriovenosa de oxigênio (Marques, 2014). Durante o exercício físico, o consumo de oxigênio (VO2) depende da integração do organismo em captá-lo, transportá-lo e utilizá-lo para produzir energia por processos aeróbios. VO2máx ocorre quando o débito cardíaco e a extração periférica de oxigênio alcançam limites que não poderão ser ultrapassados com maior carga de trabalho muscular (França et ál., 2014).

No repouso, o VO2 é similar entre indivíduos treinados e sedentários. Entretanto, durante o esforço físico máximo, os valores de $\mathrm{VO} 2$ encontrados são significativamente maiores em indivíduos treinados que em sedentários (Machado et ál., 2002).

Nesse sentido, os que praticam corrida de rua podem apresentar VO2 maior, pois realizam treino de longa duração (endurance), atividade essa que expande a capacidade cardiopulmonar e melhora o aproveitamento no consumo médio de oxigênio (Marques, 2014), permitindo a utilização do VO2máx por tempos prolongados. Por essa razão, atletas maratonistas conseguem se manter durante toda a prova entre $75 \%$ e $85 \%$ do seu VO2máx (Williams e Nute, 1983). Essa associação também é válida para outras modalidades esportivas predominantemente aeróbicas, a exemplo da natação (meio-fundo e fundo), do ciclismo e do remo (Tartuga et ál., 2008).

Mcardle et ál. (2011) afirmam que fatores como hereditariedade, treinamento, idade e gênero influenciam o VO2máx. A composição 
corporal, por sua vez, é capaz de explicar até 70\% das diferenças nos valores do VO2máx (Rocha et ál., 2011).

Diante da influência exercida pela composição corporal, o VO2máx pode ser expresso tanto de forma absoluta $(\mathrm{L} / \mathrm{min})$ quanto de forma relativa à massa corporal $\left(\mathrm{mL} \mathrm{kg}^{-1} \cdot \mathrm{min}^{-1}\right)$. Os valores relativos são usados para comparar indivíduos que diferem em índice de massa corporal (IMC), percentual de gordura (\%G) e percentual de massa corporal magra (\%MM), aspectos da morfologia que influenciam essa variável (Rocha et ál., 2011). Essa relação entre composição corporal e consumo de oxigênio é o foco do presente estudo.

\section{Metodologia}

Trata-se de uma pesquisa de campo, quantitativa, transversal e descritiva. A amostra foi composta por 36 corredores de rua, de ambos os sexos, com idade de 20 a 60 anos ( $\underline{X}=42,72 \pm 10,14$ anos $)$. As coletas ocorreram no evento FastTest, realizado em Aracaju, Sergipe, Brasil, e foram precedidas pela assinatura do termo de consentimento livre e esclarecido pelos participantes, conforme as normas de pesquisa com seres humanos determinadas na Resolução 466/2012, do Conselho Nacional de Saúde (Lei 466/2012) e na Resolução de Helsinki (World Medical Association, 2008).

\section{Avaliação antropométrica}

Para a avaliação antropométrica, foram aferidas estatura, dobras cutâneas e massa corporal total (MCT).

A realização das medidas antropométricas ocorreu em concordância com os padrões internacionais de avaliação antropométrica (Marfell-Jones et 
ál., 2012). Os dados de estatura e MCT foram coletados com os participantes em ortostase.

A MCT dos participantes foi verificada por uma balança digital adulto, com régua (Fillizola®), sensibilidade de 100 g e capacidade máxima de $200 \mathrm{~kg}$.

Para a mensuração da estatura, foi utilizado um estadiômetro fixo associado à balança (Fillizola $®)$, com sensibilidade de $1 \mathrm{~mm}$ e capacidade máxima de 2,20 $\mathrm{m}$.

O IMC foi calculado (MCT $[\mathrm{kg}] /$ estatura $[\mathrm{m}]^{2}$ ) e classificado conforme estabelece a Organização Mundial da Saúde (World Medical Association, 2000).

Por fim, as dobras cutâneas (bicipital, tricipital, subescapular, axilar, abdominal, suprailíaca, da coxa e da panturrilha) foram avaliadas com plicômetro clínico (Cescorf®, modelo Innovare) de sensibilidade de $1 \mathrm{~mm}$ e capacidade máxima de $80 \mathrm{~mm}$. Após o somatório das dobras, analisou-se o percentual de gordura e da massa magra dos corredores de rua participantes. A mensuração da circunferência de braço, coxa e panturrilha também foi realizada. A partir do protocolo de Faulkner (1968), calcularam-se os \%MM e \%G.

\section{Consumo máximo de oxigênio}

A estimativa do VO2máx foi obtida por meio da corrida/caminhada de 12 minutos de Cooper (1968). Esse teste foi utilizado com base em um estudo científico, longitudinal e transverso realizado por Kennedy Cooper, em 1968. A finalidade do procedimento é avaliar o consumo máximo de oxigênio naqueles que realizaram sua aplicação. Os sujeitos percorreram a máxima distância possível no tempo predeterminado, e a distância máxima alcançada ao final do teste foi calculada. 
A partir das metragens percorridas individualmente, foi calculado o VO2máx predito, tendo como base a fórmula proposta por Cooper (1968), que leva em conta a máxima distância percorrida (DP): VO2máx (mL kg1.min-1 $)=(m-504,9) / 44,73]$, em que "m" é a distância atingida pelo indivíduo. Tanto a pressão arterial quanto a frequência cardíaca foram mensuradas anteriormente ao teste.

É válido ressaltar que o procedimento foi interrompido com a exaustão voluntária do indivíduo, sendo a distância total utilizada para critérios de avaliação.

\section{Análise estatística}

Os dados foram organizados e planilhados por meio do programa Microsoft Office Excel $®$ 2016. Após essa etapa, realizou-se uma análise de normalidade dos dados através do D’agostino \& Pearson Omnibus Normality Test para cada variável independente, sendo que a variável idade se encontrou dentro da normalidade. Após a identificação da normalidade, observou-se que os dados de idade, \%G e \%MM foram paramétricos, sendo realizado o teste de correlação de Pearson para p <0,05 entre as variáveis VO2máx e idade de todos os sujeitos, bem como entre grupos masculino e feminino, respectivamente. A variável IMC não apresentou normalidade nos testes e foi processada por meio do teste de correlação de Spearman para dados não paramétricos.

\section{Resultados e discussão}

Participaram do estudo 36 corredores de rua, dos quais 21 eram homens e 15, mulheres, com idade mínima de 20 anos e máxima de 60 anos ( $\underline{X}=$ $42,72 \pm 10,14$ anos). 
Na análise de idade e VO2máx, não houve correlação entre essas variáveis (tabela 1), apresentando coeficiente de correlação de Pearson $r=-0,27$, cujo valor, segundo Callegari-Jacques (2009), é fraco.

Quando discriminado por gênero, o resultado se manteve para o gênero feminino $(r=-0,17)$, enquanto no gênero masculino, houve moderada correlação negativa entre as variáveis $(\mathrm{r}=-0,66)$. No intragrupo masculino, portanto, notou-se que quanto maior é a idade, menor será o VO2máx ou vice-versa.

Tabela 1. Correlação do VO2máx com as variáveis idade e IMC em homens e mulheres

\begin{tabular}{|c|c|c|c|c|c|c|}
\hline \multirow{3}{*}{ Variáveis } & \multicolumn{6}{|c|}{ V02 máx (mL.Kg $\left.{ }^{-1} \cdot \min ^{-1}\right)$} \\
\hline & \multicolumn{2}{|c|}{ Geral } & \multicolumn{2}{|c|}{ Homens } & \multicolumn{2}{|c|}{ Mulheres } \\
\hline & $r$ & $\mathrm{p}$ & $r$ & $\mathrm{p}$ & $\mathrm{r}$ & $\mathrm{p}$ \\
\hline Idade (anos) & $-0,27$ & 0,051 & $-0,66$ & 0,0003 & $-0,17$ & 0,406 \\
\hline IMC $\left(\mathrm{kg} / \mathrm{m}^{2}\right)$ & $-0,66$ & 0,0001 & $-0,79$ & 0,0001 & $-0,63$ & 0,0005 \\
\hline
\end{tabular}

Fonte: elaboração própria.

A correlação das variáveis IMC x VO2máx (tabela 1), sem distinção de gênero, foi negativa moderada $(r=-0,66)$. Da maneira similar, foi possível observar forte correlação negativa na análise intragrupo masculino e moderada correlação intragrupo feminino, com $r=-0,79$ e $r=-0,63$, respectivamente. Diante disso, evidenciou-se que maiores valores de IMC implicam menor VO2 máx.

O estudo da relação entre as variáveis \%G x VO2máx mostrou forte correlação negativa, coeficiente de correlação de Pearson $r=-0,76$ (figura 1). Da mesma forma, foi possível observar forte correlação negativa na análise intragrupo masculino e moderada no intragrupo feminino com $\mathrm{r}=-0,74$ e $\mathrm{r}=-0,60$, respectivamente. Assim, quanto maior a $\% \mathrm{G}$, menor o VO2 máx ou vice-versa. 
Figura 1. Correlação entre percentual de gordura e consumo máximo de oxigênio

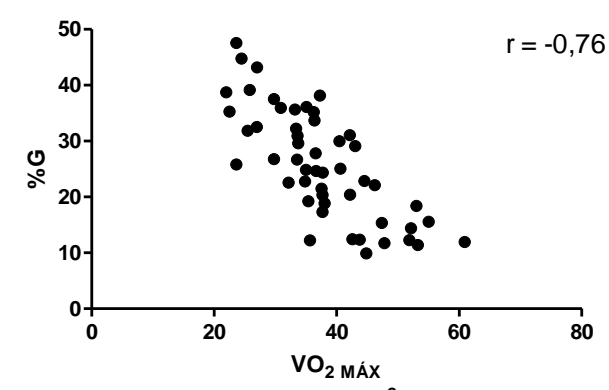

1A)

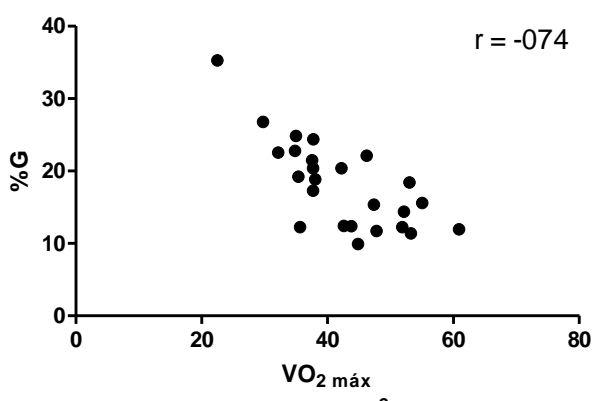

1B)

1C)

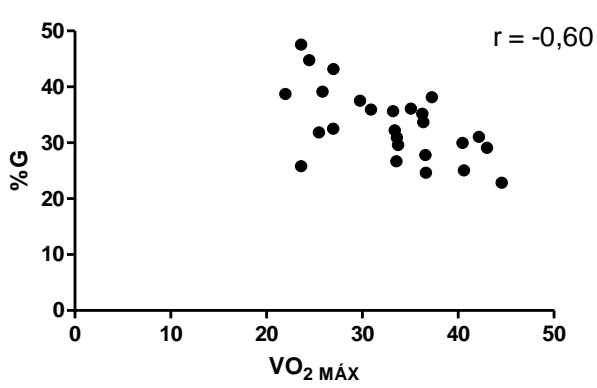

$\left(\mathrm{ml}^{\prime} \mathrm{kg} \cdot \mathrm{min}^{-2}\right)$

1A): todos os indivíduos do estudo representados; 1B): apenas o gênero masculino; 1C): apenas o gênero feminino.

Fonte: elaboração própria.

Ao correlacionar as variáveis \%MM x VO2máx, notou-se forte correlação positiva, coeficiente de correlação de Pearson $r=0,76$. Semelhantemente, foi possível observar forte correlação positiva na análise do grupo masculino e moderada do grupo feminino, com $r=0,74$ e $r=0,60$, respectivamente. Esses resultados indicam que, quanto maior a \%MM, maior o VO2máx ou vice-versa. 
Figura 2. Correlação entre o percentual de massa magra e o consumo máximo de oxigênio

2A)
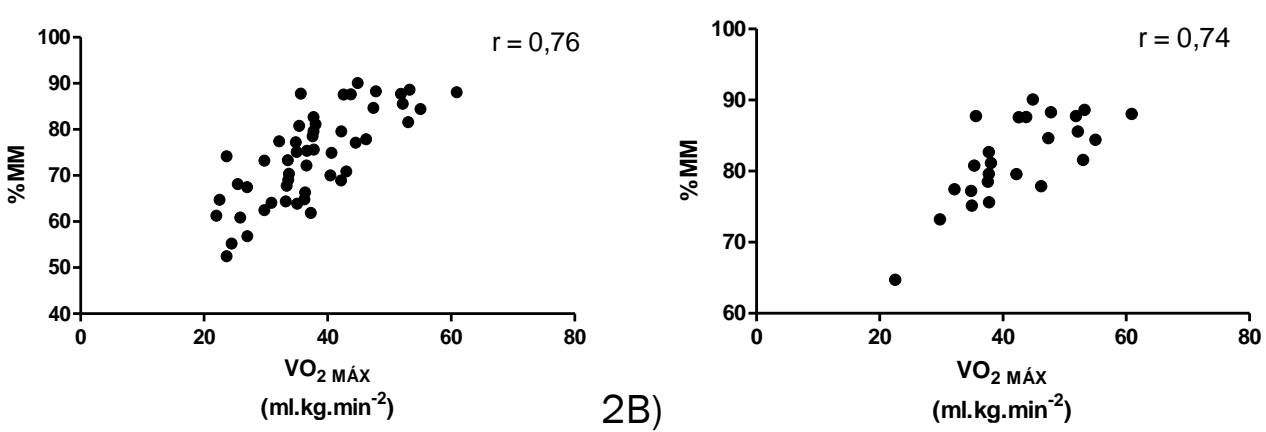

2C)

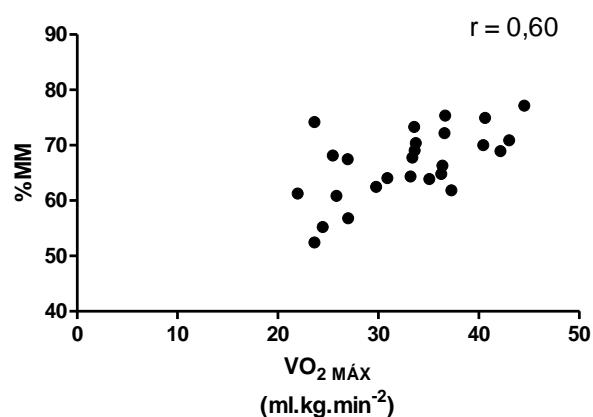

2A): todos os indivíduos do estudo representados; 2B): apenas o gênero masculino, 2C): apenas o gênero feminino.

Fonte: elaboração própria.

Apesar de o presente estudo não encontrar correlação entre as variáveis idade e $\mathrm{VO} 2$, a literatura aponta relação negativa significativa. Hawkins e Wiswell (2003) e Silva et ál. (2016) encontraram que o consumo máximo de oxigênio declina com o avançar da idade. Entre as explicações, está a redução da funcionalidade fisiológica com o envelhecimento, o que implica menos atividades físicas e consequente diminuição da capacidade cardiorrespiratória, mesmo em atletas (Arantes et ál., 2017). O principal achado do presente estudo, entretanto, foi a influência da composição corporal sobre a VO2máx dos praticantes de corrida de rua.

Houve forte correlação negativa entre VO2máx e \%G, semelhantemente aos achados de estudos anteriores realizados tanto em corredores de rua 
como em atletas de outras modalidades aeróbicas. Waclawovsky et ál. (2018) encontraram relação inversa $(r=-0,655$; $p<0,001)$ entre \%G e VO2máx em corredores de rua; Pancotto et ál. (2015) identificaram relação também negativa, embora pequena, entre esses parâmetros em atletas de futebol da categoria juniores; Regert et ál. (2014) apontaram resultados similares em duas equipes de futebol do Rio Grande do Sul. Detoni et ál. (2015) e Sousa et ál. (2018) apontam que sujeitos sedentários e com gordura corporal elevada apresentam desempenho físico reduzido resultante da diminuição na capacidade cardiorrespiratória. Diante disso, atletas de endurance com melhor performance apresentam medidas baixas de dobra cutânea, bem como menor peso (Milagre, 2015).

Diferentemente do \%G, a \%MM apresentou forte correlação positiva com a VO2máx, como também demonstrado em Waclawovsky et ál. (2018). A \%MM é um descritor razoável e aceitável da capacidade metabólica máxima dos músculos e, consequentemente, do rendimento em provas de corrida (Gorla et ál, 2017; Filipe, 2018; Barrientos et ál., 2020). Em contrapartida, Santi et ál. (2018) encontraram em seu estudo que obesos praticantes de corrida e obesos sedentários não diferiram significativamente em \%MM, mas diferiram em VO2máx, o que indica que a massa magra estaria mais relacionada à força muscular que à capacidade cardiorrespiratória.

Quando discriminada por gênero, a relação positiva entre VO2máx e \%MM se manteve forte para o grupo masculino, mas apenas moderada no intragrupo feminino, em concordância com Waclawovsky et ál. (2018), que, de forma similar, não encontraram relação significativa em mulheres. Esse fenômeno é explicado pelo maior volume de massa muscular no grupo masculino, sendo esse um dos componentes da massa corporal magra, a qual também inclui água, tecidos conjuntivo, ossos e órgãos internos. É importante lembrar que a massa muscular demanda maior 
quantidade de oxigênio quando comparada ao tecido adiposo (Waclawovsky et ál., 2018; Yoshiga e Higuchi, 2003). O menor volume de hemácias descrito para o grupo feminino é outra explicação possível para essa diferença entre gêneros (Waclawovsky et ál., 2018).

A correlação negativa entre \%G e VO2máx encontrada foi forte para o intragrupo masculino, mas moderada para o feminino, em consonância com Waclawovsky et ál. (2018), e pode ser explicada pela diferença em proporção de massa magra, metabolicamente mais ativa, já descrita entre os grupos.

No que tange ao IMC, a moderada correlação negativa entre esse índice e o VO2máx concorda com estudos anteriores como Conte et ál. (2003), que avaliaram indivíduos em uma pista de caminhada em Sorocaba; Tartaruga et ál. (2008), cujo estudo teve remadores e corredores de rendimento como amostra. Santi (2018) identificou que, apesar de os corredores com IMC elevado terem VO2máx maior que os indivíduos sedentários com IMC elevado, eles apresentam VO2 máx $[42,5(8,0) \mathrm{mL} / \mathrm{kg} / \mathrm{min}]$ inferior aos corredores de peso normal [VO2máx: 49,2 $(1,8) \mathrm{mL} / \mathrm{kg} / \mathrm{min}$ ], o que reforça a correlação negativa entre IMC e capacidade cardiorrespiratória. Diante disso, Rodrigues e Padovani (2015) sugerem que o IMC dos maratonistas deve se situar entre 17,5 e 20,7 Kg/m2, considerando 19,8 $\mathrm{Kg} / \mathrm{m} 2$ o valor ótimo entre para melhor desempenho masculino.

Na discriminação entre gêneros, a relação IMC x VO2máx apresentou forte relação negativa apenas no sexo masculino. Nesse sentido, alguns autores relatam que a massa adiposa em níveis elevados subestima os valores de VO2máx quando este é corrigido pela MCT (Tartaruga et ál., 2008; Rodrigues e Padovani, 2015; Toth et ál., 1993; Tolfrey et ál., 2006; Winter e Hamley, 1976), uma vez que a maior parte do oxigênio é direcionada ao músculo esquelético durante o exercício (Waclawovsky et ál., 2018). 
Assim, como a composição corporal feminina apresenta maior \%G

(Waclawovsky et ál., 2018; Silva et ál., 2017), tecido com menor demanda de oxigênio, o aumento do IMC estaria menos relacionado com o aumento da VO2máx, quando comparado ao grupo masculino.

Por essas razões, Hermann (2019) reforça que a composição corporal é melhor preditor de desempenho, em especial da velocidade de corrida, que o IMC, visto que diferencia massa livre de gordura de massa gorda e reflete o nível de atividade física do indivíduo.

\section{Conclusões}

Com base nos resultados descritos, conclui-se que, no grupo de corredores de rua, não foi encontrada correlação entre a idade e a capacidade cardiorrespiratória, exceto para o grupo masculino, no qual houve correlação negativa moderada. O IMC e \%G foram preditores negativos de VO2máx, com correlação moderada e forte, respectivamente. O \%MM apresentou forte correlação positiva com capacidade cardiorrespiratória, principalmente no grupo masculino. Diante disso, fica evidente que o estudo da composição corporal tem papel essencial no treinamento para modalidades aeróbicas e desempenho em provas de endurance.

\section{Referências}

Arantes, F. J., Vieira, P.F., Borges, D. L. e Pereira, A. A. (2017). Pode o consumo máximo de oxigênio e a frequência cardíaca máxima medidos em teste laboratorial serem preditos por equações em corredores amadores? RBPFEX-Revista Brasileira de Prescrição e Fisiologia do Exercício, 11(66), 343-352. https://doi.org/10.33233/rbfe.v11i2.3385

Barrientos, G., Alves, J., Toro, V., Robles, M. C., Muñoz, D. e Maynar, M. (2020). Association between trace elements and body composition parameters in endurance runners. International Journal of Environmental Research and Public Health, 17(18), 6563. https://doi.org/10.3390/ijerph17186563 
Callegari-Jacques, S. M. (2009). Bioestatística: princípios e aplicações. Artmed.

Conte, M., Domingues, S. P. de T., Godoi, V. J. de, Más, E. F., Vazatta, R. e Teixeira, L. F. M. (2003). Interação entre VO2máx, índice de massa corporal e flexibilidade. Revista Mackenzie de Educação Física e Esporte, 2(2), 23-30. https://doi.org/10.1590/s1807-55092013005000005

Cooper, K. H. (1968). A means of assessing maximal oxygen intake: Correlation between field and treadmill testing. Jama, 203(3), 201-204. https://doi.org/10.1001/jama.1968.03140030033008

Detoni, G. C., Oliveira, V. M. de, Ferreira, C., Queiroga, M. R., Peyré-Tartaruga, L. A. e Tartaruga, M. P. (2015). Influência do modelo alométrico na relação entre consumo máximo de oxigênio e desempenho de corredores fundistas. Revista Brasileira de Ciências do Esporte, 37(4), 389-394. https://doi.org/10.1016/j.rbce.2015.08.012

Faulkner, J. A. (1968). Physiology of swimming and diving. Em H. Falls (org.), Exercise physiology (pp. p.415-446). Academic Press.

Ferreira, V. R., Bento, A. P. N. e Silva, M. R. (2015). Consumo alimentar, perfil antropométrico e conhecimentos em nutrição de corredores de rua. Revista Brasileira de Medicina do Esporte, 21(6), 457-461. https://doi.org/10.1590/1517$\underline{869220152106138411}$

Filipe, D. (2018). Condição cardiorrespiratória e regulação autonómica cardíaca: estudo da importância da composição corporal. (Tese de doutorado). Instituto Politécnico de Santarém, Rio Maior, Portugal. https://repositorio.ipsantarem.pt/handle/10400.15/2493

França, E. de, Caperuto, E. C. e Hirota, V. B. (2014). Testes indiretos de VO2 máximo devem ser escolhidos de acordo com o gênero, variáveis antropométricas e capacidade aeróbica presumida. Revista Brasileira de Prescrição e Fisiologia do Exercício, 8(49), 712-721. https://doi.org/10.33233/rbfe.v11i2.3387

Herrmann, F. R., Graf, C., Karsegard, V. L., Mareschal, J., Achamrah, N., Delsoglio, M., Schindler, M., Pichard, C. e Genton, L. (2019). Running performance in a timed city run and body composition: A cross-sectional study in more than 3000 runners. Nutrition, 61, 1-7. https://doi.org/10.1016/j.nut.2018.10.022

Revista de Investigación Cuerpo, Cultura y Movimiento ISSN: 2248-4418 | e-ISSN: 2422-474X | DOI: https://doi.org/10.15332/2422474X Vol. 11 N. 02 | julio-diciembre de 2021 
Gorla, J. I., Silva, A. de A. C., Campos, L. F. C. C. de, Santos, C. F. dos, Almeida, J. J. G. de, Duarte, E. e Queiroga, M. R. (2017). Composição corporal e perfil somatotípico de atletas da seleção brasileira de futebol de 5. Revista Brasileira de Ciências do Esporte, 39(1), 79-84. https://doi.org/10.1016/j.rbce.2015.12.016

Hawkins, S. e Wiswell, R. (2003). Rate and mechanism of maximal oxygen consumption decline with aging: implications for exercise training. Sports Med, 33(12), 877-888. https://doi.org/10.2165/00007256-200333120-00002

Lei 466, 12 de dezembro de 2012 (2012). Pela qual são expedidas as normas para realizar investigações em seres humanos. https://bvsms.saude.gov.br/bvs/saudelegis/cns/2013/reso466 $12 \quad 12$ 2012.html

Machado, F. A., Guglielmo, L. G. A. e Denadai, B. S. (2002). Velocidade de corrida associada ao consumo máximo de oxigênio em meninos de 10 a 15 anos. Revista Brasileira de Medicina do Esporte, 8(1), 1-6. https://doi.org/10.1590/s1517$\underline{86922002000100001}$

Marfell-Jones, M. J., Stewart, A. D. e De Ridder, J. H. (2012). International standards for anthropometric assessment. International Society for the Advancement of Kinanthropometry.

Marques, V. A. (2014). A influência do treinamento de força para a melhoria do VO2 máximo dos corredores de rua. FIEP Bulletin On-line. http://www.fiepbulletin.net/index.php/fiepbulletin/article/view/4332/8474

McArdle, W. D., Katch, F. I. e Katch, V. L. (2011). Fisiologia do exercício: nutrição, energia e desempenho humano. Guanabara Koogan.

Milagre, E. D. (2015). Comparação das respostas cardiorrespiratórias entre corredores capixabas de diferentes níveis de desempenho. (Dissertação de mestrado). Universidade Federal do Espírito Santo, Vitória, Espírito Santo, Brasil. https://doi.org/10.22564/4simbgf2010.194

Pancotto, F. A., Jr., Crescente, L. A. B., Cardoso, M. e Siqueira, O. D. (2015, 19 jun.). VO2 máximo e composição corporal em atletas de futebol da categoria juniors. EFDeportes.com. https://www.efdeportes.com/efd151/vo2-maximo-em-atletasde-futebol.htm\#: :text=Os\%2oresultados\%20obtidos\%20atrav\%C3\%A9 s\%20da, $51 \% 25 \% 20 \mathrm{e} \% 2056 \% 2 \mathrm{Co} 3$

Revista de Investigación Cuerpo, Cultura y Movimiento ISSN: 2248-4418 | e-ISSN: 2422-474X | DOI: https://doi.org/10.15332/2422474X Vol. 11 N.o 2 | julio-diciembre de 2021 
Perez, A. J., Milagre, E. D., Carletti, L., Gomes, K. B., Lourenço, T. F. e Fortes, L. S. (2004). Perfil cardiopulmonar de corredores de rua atletas e não atletas caracterizados por nível de desempenho esportivo. Revista Brasileira de Ciência e Movimento, 26(1), 105-115. https://doi.org/10.11606/d.100.2017.tde-06042017175218

Regert, P. L., Koehler, C., Reuter, E. M., Pohl, H. H. e Reckziegel, M. B. (2014). Percentual de gordura e consumo máximo de oxigênio: uma associação de variáveis de aptidão física de atletas de futebol. FIEP Bulletin On-line.

https://doi.org/10.33233/rbfe.v12i1.3311

Rocha, P. C., Goulart, G. A., Ferreira, F. O., Lima, M. M., Amorin, F. T. e Peixoto, M. F. (2011). Relação entre o percentual de gordura e a capacidade aeróbia máxima em militares do $3^{\circ}$ batalhão da polícia militar de minas gerais da cidade de diamantina. Revista Brasileira de Prescrição e Fisiologia do Exercício, 5(29), 9. https://doi.org/10.33233/rbfe.v10i3.3437

Rodrigues, C. de A. e Padovani, L. Z. (2015, 19 jun.). Maratonistas: aspectos físicos envolvidos na prescrição do treino. EFDeportes.com.

https://doi.org/10.11606/d.47.2008.tde-16022009-153544

Santi, A., Bosch, T. A., Bantle, A. E., Alvear, A., Wang, Q., Hodges, J. S., Dengel, D. R. e Chow, L. S. (2018). High body mass index masks body composition differences in physically active versus sedentary participants. Metabolic syndrome and related disorders, 16(9), 483-489. https://doi.org/10.1089/met.2018.0042

Silva, C. G. de S., Franklin, B. A., Forman, D. E. e Araújo, C. G. S. (2016). Influence of age in estimating maximal oxygen uptake. Journal of Geriatric Cardiology: JGC, 13(2), 126. https://doi.org/10.11909/j.issn.1671-5411.2016.02.010

Silva, R. da, Jr., Abreu, W. C. de e Silva, R. F. da (2017). Composição corporal, consumo alimentar e hidratação de praticantes de musculação. RBNE - Revista Brasileira de Nutrição Esportiva, 11(68), 936-946.

https://doi.org/10.22533/at.ed.5152003126

Sousa, S., Andrade, E. e Marangoni, M. A. (2018). Relações entre potência muscular e composição corporal. RBPFEX - Revista Brasileira de Prescrição e Fisiologia do Exercício, 12(79), 1045-1051.

http://www.rbpfex.com.br/index.php/rbpfex/article/view/1574

Revista de Investigación Cuerpo, Cultura y Movimiento ISSN: 2248-4418 | e-ISSN: 2422-474X | DOI: https://doi.org/10.15332/2422474X Vol. 11 N.o 2 | julio-diciembre de 2021 
Tartaruga, M. P., Belike, D. D., Coertjens, M., Baptista, R. R., Tartaruga, L. A. P. e Kruel, L. F. M. (2008). Relação entre consumo máximo e submáximo de oxigênio em corredores e remadores de rendimento. Journal of Physical Education, 77(141), 22-33. https://revistadeeducacaofisica.emnuvens.com.br/revista/article/view/39o

Tolfrey, K., Barker, A., Thom, J. M., Morse, C. I., Narici, M. V. e Batterham, A. M. (2006). Scaling of maximal oxygen uptake by lower leg muscle volume in boys and men. Appl Physiol, 10o(6), 1851-1856. https://doi.org/10.1152/japplphysiol.01213.2005

Toth, M. J., Goran, M.I., Ades, P. A., Howard, D.B. e Poehlman, E. T. (1993). Examination of data normalization procedures for expressing peak $\mathrm{VO} 2$ data. J Appl Physiol, 75(5), 2288-2292. https://doi.org/10.1152/jappl.1993.75.5.2288

Waclawovsky, G., Silva, L. R. da Vidaletti, D., Ferrari, R. e Lehnen, A. M. (2018). Associação da composição corporal e capacidade cardiorrespiratória em corredores de rua. RBPFEX-Revista Brasileira de Prescrição e Fisiologia do Exercício, 12(80), 1138-1148. https://doi.org/10.33233/rbfe.v5i1.3554

Williams, C. e Nute, M. L. (1983). Some physiological demands of a half-marathon race on recreational runners. British Journal of Sports Medicine, 17(3), 152-161. https://doi.org/10.1136/bjsm.17.3.152

Winter, E. M. e Hamley E. J. (1976). Sub maximal oxygen uptake related to fat free mass and lean leg volume in trained runners. British Journal of Sports Medicine, 10(4), 223-225. https://doi.org/10.1136/bjsm.10.4.223

World Health Organization. (2000). Obesity: preventing and managing the global epidemic. World Health Organization. https://apps.who.int/iris/handle/10665/63854

World Medical Association. (2008). Declaration of Helsinki: Ethical principles for medical research involving human subjects.

https://doi.org/10.1515/9783110208856.233

Yoshiga, C. C. e Higuchi, M. (2003). Oxygen uptake and ventilation during rowing and running in females and males. Scand $J$ Med Sci Sports, 13(6), 359-363.

https://doi.org/10.1046/j.1600-0838.2003.00324.x

Revista de Investigación Cuerpo, Cultura y Movimiento ISSN: 2248-4418 | e-ISSN: 2422-474X | DOI: https://doi.org/10.15332/2422474X Vol. 11 N.o 2 | julio-diciembre de 2021 